\title{
A Survey of Canine Anaesthesia in Veterinary Practice in Cluj-Napoca
}

\author{
Cosmin PESTEAN ${ }^{1 *}$, Liviu OANA ${ }^{1}$, Lucia BEL ${ }^{1}$, Iuliu SCURTU ${ }^{1}$, George CLINCIU ${ }^{2}$, Ciprian OBER ${ }^{1}$ \\ ${ }^{1}$ Faculty of Veterinary Medicine, University of Agricultural Sciences and Veterinary Medicine, 400372 \\ Cluj-Napoca, Mănăştur Street 3-5, Romania \\ ${ }^{2}$ Creative Zone-Vet SRL, 507055 Cristian Brasov, Teilor Street 3, Romania \\ *corresponding author: cosmin.pestean@usamvcluj.ro
}

Bulletin UASVM Veterinary Medicine 73(2) / 2016,

Print ISSN 1843-5270; Electronic ISSN 1843-5378

DOI:10.15835/buasvmcn-vm: 12192

\begin{abstract}
A survey with 32 questions was performed in Cluj-Napoca veterinary private practices about the routine anaesthetic management of dogs. Of those veterinarians who answered this questionnaire, $18.2 \%$ are equipped with a machine for inhalation anesthesia, $27.3 \%$ do not use any monitoring during anesthesia and no one use methods of monitoring blood pressure. All the veterinarians graduated the Faculty of Veterinary Medicine Cluj-Napoca. Ketamine and alpha 2 agonists remain the most used anesthetic substances in private practices. Postoperative analgesia is used constantly by $81.8 \%$ of veterinarians. The mortality rate in veterinary practices in Cluj - Napoca for two years was $0.25 \%$ (1:403).
\end{abstract}

Keywords: anaesthesia, monitoring, private practices, survey

\section{INTRODUCTION}

The ability to restrain animals using a drug or drug combinations plays a central role in veterinary practice enabling veterinarians to make animals more tractable for examination, to provide general anesthesia for surgery and to provide analgesia when needed (Nicholson and Watson, 2001).

Veterinary anesthesia and analgesia is one of the areas of veterinary medicine with remarkable progress in recent decades, benefiting from the emergence of new anesthetic agents, protocols and improved monitoring technologies, representing a challenge for veterinarians who have to cope with this progress in veterinary health care.

The anesthesia caseload at most veterinary college teaching hospitals has become increasingly challenging, complicated, and timeconsuming. Many of the animals anesthetized at veterinary college hospitals have chronic illnesses or conditions that may affect their responses to anesthetic drugs, whereas many private practices deal mainly with young healthy animals undergoing elective procedures such as ovariohysterectomy and castration. One concern related to these trends is that the anesthesia training offered to veterinary students may not be applicable to the types of animals and procedures requiring anesthesia at most private veterinary practices (Wagner and Hellyer, 2000).

To have factual data about how anesthesia is performed in veterinary practices in Cluj-Napoca, we have developed a questionnaire that was presented to veterinarians in private cabinets existing in this city.

\section{MATERIALS AND METHODS}

A questionnaire (a copy can be viewed on request from the corresponding author) divided into four parts with a total of 32 questions was presented and explained during April -May 2014 to a total of 24 veterinarians (owners of cabinets 
and private clinics or freelancers). The first part of the questionnaire consists of nine questions about the structure and organization of veterinary activity. The second part contains questions about the existence of specialized equipment, elements of difficulty in terms of achieving anesthesia but also questions about satisfaction of veterinarians from private sector regarding the training of the students or graduates in the field of veterinary anesthesia. Part three, the most consistent one, with 16 questions, relates to substances and protocols used in canine anesthesia and analgesia. The last part addresses the issue of complications and incidents occurring during canine anesthesia. Each respondent was assured that the answers would remain anonymous.

\section{RESULTS AND DISCUSSIONS}

Of the 24 veterinarians approached, 22 responded positively and completely to this study. All these are graduates of the Faculty of Veterinary Medicine Cluj-Napoca, $72.7 \%$ are male and $90.9 \%$ deals exclusively with small animals. The most common form of organization of the veterinary activity was private practice - $72.7 \%, 9.1 \%$ as veterinary clinic and $4.6 \%$ freelancer.

Regarding the questions of the second part, which refers to the equipment used, the results are very interesting. Among the veterinarians surveyed, $31.9 \%$ answered that they have a source of oxygen and $18.2 \%$ are equipped with a machine for inhalation anesthesia. By comparison, 93.8\% of South African veterinarians are equipped with inhalational anesthesia apparatus (Joubert, 2000). Anesthesia monitoring is very important and allows early detection of problems associated with anesthesia. Of respondents, $27.3 \%$ do not use any monitoring during anesthesia, in comparison, $31.3 \%$ of French veterinarians and $7 \%$ of Australians do not use any method of monitoring canine anesthesia (Farges 2012; Nicholson and Watson, 2001). A large percentage of veterinarians monitor temperature $72.7 \%$, ECG and pulse oximetry $40.1 \%$, apnea monitor $9.1 \%$ and $4.6 \%$ capnography. None of the veterinarians surveyed do not use methods of monitoring blood pressure. The existence of important additional equipment for anesthesia is: endotracheal tubes and heating devices 77,3\%, Ambu bag 59,1\%, face mask $40,1 \%$, syringe pump $31,9 \%$, infusomat $22,7 \%$ and ventilator $27,3 \%$. In terms of staff training,
$68.1 \%$ are able to monitor anesthesia and $63.6 \%$ to perform resuscitation maneuvers. From the respondents, $18.2 \%$ have trouble knowing how to use the anesthesia and ventilation apparatus while $4.6 \%$ have problems regarding resuscitation and intubation, $13.6 \%$ other issues. Knowledge of anesthetic substances, interpretation of monitoring data, placing an intravenous catheter or fluidotherapy do not raises any problems with any of those interviewed. From those surveyed $31.9 \%$ believes that graduates of the Faculty of Veterinary Medicine Cluj-Napoca are not well prepared regarding anesthesia, 31.9\% that are well prepared and the remaining $36.4 \%$ have no opinion about this. Regarding the opportunity for a postgraduate training in veterinary anesthesia and resuscitation, $59.1 \%$ responded positively to this question, $13.6 \%$ negative and the remaining $27.3 \%$ have no opinion.

The third part of this study was dedicated to canine anesthesia. Number of anesthetized dogs every month at the clinics in Cluj-Napoca was between 3 and 100, the average of anesthetized dogs / clinic / month is 18.3 . At $21.2 \%$ of dogs anesthesia was conducted for a more effective clinical examination, the other dogs needing surgery. Intravenous catheters were used in $72.7 \%$ of convenience cases and $81.8 \%$ of patients undergoing major surgery. In a similar study in France the intravenous catheters for elective cases are used in a proportion of $72.3 \%$ and $95.2 \%$ for major cases (Farges, 2012). Regarding the use of endotracheal tubes, $18.2 \%$ of those surveyed always use them and $59.1 \%$ use them occasionally for convenience surgery, $22.7 \%$ do not use this method. In major cases $45 \%$ use endotracheal tubes, $40.9 \%$ sometimes and $13.6 \%$ do not use atall. A study from 2000 conducted on a representative batch of American vets show that $95 \%$ of them always use endotracheal tubes (Wagner and Hellyer, 2000). Intravenous administration of infusions solutions to convenience cases is performed in a proportion of $72.7 \%$ and only $18.2 \%$ occasionally. Intravenous solutions are regularly administered in $86.3 \%$ cases of major surgery and sometimes $4.6 \%$. Veterinarians use premedication separate from anesthesia induction, in proportion of $59.1 \%$ of cases. The most commonly used anesthetic substances are presented in Table 1.

It is observed that ketamine and alpha 2 agonists remain the most used substances, but also 
Tab. 1 Anesthetic substances used for canine general anesthesia

\begin{tabular}{ccc}
\hline Substance & Convenience surgery & Major surgery \\
\hline xylazine & $95,4 \%$ & $86,4 \%$ \\
ketamine & $95,4 \%$ & $81,8 \%$ \\
atropine & $77,3 \%$ & $81,8 \%$ \\
diazepam & $77,3 \%$ & $68,1 \%$ \\
medetomidine & $54,5 \%$ & $40,1 \%$ \\
propofol & $50 \%$ & $36,4 \%$ \\
acepromazine & $36,4 \%$ & $31,9 \%$ \\
butorphanol & $27,3 \%$ & $31,9 \%$ \\
midazolame & $22,7 \%$ & $18,2 \%$ \\
isoflurane & $18,2 \%$ & $18,2 \%$ \\
dexmedetomidine & $13,6 \%$ & $13,6 \%$ \\
halotane & $9,1 \%$ & $9,1 \%$ \\
fentanyl & $9,1 \%$ & $4,6 \%$ \\
morphine & $9,1 \%$ & $4,6 \%$ \\
thiopental & $4,6 \%$ & $4,6 \%$ \\
zolazepam/tiletamine & $4,6 \%$ & $4,6 \%$ \\
sevoflurane & $0 \%$ & $0 \%$ \\
alfaxalone & $0 \%$ & $0 \%$ \\
etomidate & $0 \%$ & $0 \%$ \\
buprenorphine & $0 \%$ & $0 \%$ \\
romifidine & $0 \%$ & $0 \%$ \\
glycopyrrolate & $0 \%$ & $0 \%$ \\
other & $9,1 \%$ & $9,1 \%$ \\
\hline
\end{tabular}

that propofol is becoming increasingly popular in private practices.

Veterinarians use pre/intraoperatively analgesia for $63.6 \%$ of convenience surgeries, and $72.7 \%$ of major surgical cases. Of those which actually use pre/intraoperative analgesia for convenience interventions, the most used substance is ketamine $100 \%$, followed by NSAIDs 92.9\%, butorphanol $42.9 \%$, tramadol $42.9 \%$ and others $42.9 \%$. In major cases the molecules used are: ketamine $100 \%$, NSAIDs $87.5 \%$, tramadol $62.5 \%$, butorphanol $43.8 \%$, (dex) medetomidine $31.2 \%$, fentanyl $12.5 \%$, morphine $6,3 \%$, and others $31.2 \%$. In both surgeries for convenience and for major cases, $77.3 \%$ of surveyed veterinarians declare that they use loco-regional anesthesia. Postoperative analgesia is used constantly by $81.8 \%$ of veterinarians and in major cases the percentage reaches $86.4 \%$. Even if across all studies those surveyed accept the importance of analgesia, a relatively small number of them always uses analgesic substances in the perioperative period, 22\% (Lascelles, 1999) and 30\% (West et al., 2009).
The last part of this study was designed to complications associated with anesthesia, accidents and mortality cases associated with general anesthesia. The most frequent complications that veterinarians in Cluj - Napoca encounter during anesthesia are: awakening during anesthesia 77.3\%, apnea $72.7 \%$, hypoventilation $63.6 \%$, prolonged recovery $59.1 \%$, bradycardia $54,5 \%$, dyspnea $50 \%$, arrhythmia $50 \%$, hypotension $27.3 \%$, tachycardia $22.7 \%$ and hypertension $9.1 \%$. A number of 24 mortalities were associated with general anesthesia in the past two years in surveyed veterinary clinics. By extrapolating the results presented in the third part, the total number of anesthetized dogs / two years is approximately 9672. In consequence, mortality rate in veterinary practices in Cluj Napoca for two years is $0.25 \%$ ( 1 death per 403 anesthetized dogs). This percentage is high if we compare with other, older studies, were the ratio is 1:679 (Clarke and Hall, 1990) and 1:1243 (Joubert, 2000). Cardio respiratory resuscitation technique has been used successfully by $77.3 \%$ of surveyed veterinarians. 


\section{CONCLUSIONS}

This study was conducted exclusively in ClujNapoca in order to achieve later a comparison between performance of canine anesthesia in a relatively small area but benefiting from two different systems: teaching hospital and private practices. The lack of standard protocols for implementation and monitoring of veterinary anesthesia creates discrepancies between private offices but especially between them and the teaching hospital. Students might be less prepared to face practical and economic realities of routine private practice. Perioperative IV administration of fluids, blood pressure monitoring, and postoperative pain management are not consistently provided, thus making evident that there is room for improvement in the standard of veterinary care in private practices.

\section{REFERENCES}

1. Capner CA, Lascelles BDX, Waterman-Pearson AE (1999). Current British veterinary attitudes to perioperative analgesia for dogs. Vet Rec. 145(4): 95-99.

2. Clarke KW, Hall LW (1990). A survey of anaesthesia in small animal practice: AVA/BSAVA report. Veterinary Anaesthesia and Analgesia 17(1): 4-10.
3. Farges C (2012). Etude sur la pratique et les moyens mis en oeuvre pour l'anesthesie des chiens - resultats d'une enquete en ligne aupres des veterinaires praticiens français. Vetagro Sup, Campus veterinaire de Lyon.

4. Joubert KE (2000). Routine veterinary anaesthetic management practices in SouthAfrica. Journal of the South African Veterinary Association 71(3): 166-172.

5. Lascelles BDX, Capner CA, Waterman-Pearson AE (1999). Current British veterinary attitudes to perioperative analgesia for cats and small mammals. Vet Rec 145:601604.

6. Nicholson A, Watson ADJ (2001). Survey on small animal anaesthesia. Australian Veterinary Journal 79(9): 613619.

7. Wagner AE, Hellyer PW (2000). Survey of anesthesia techniques and concerns in private veterinary practice. JAVMA 217(11): 1652 - 1657.

8. West E, Anreoni V, Keeley BJ, Self IA, Jones BR (2009). Analgesic management of an eight-year-old Springer Spaniel after amputation of a thoracic limb. Ir Vet J 62(2): 111-118.

9. Williams VM, Lascelles BDX, Robson MC (2005). Current attitudes to, and use of, peri-operative analgesia in dogs and cats by veterinarians in New Zealand. New Zealand Veterinary Journal 53(3): 193-202. 